\title{
Modeling of the Viscosity Behavior in the Interface Area of Two Miscible Liquids
}

\author{
E. Szwajczak ${ }^{a}$, R. Stragaczyński ${ }^{a}$, H. Herba ${ }^{a}$, J. ŚWiergiel ${ }^{b, *}$ And J. JAdŻyN ${ }^{b}$ \\ ${ }^{a}$ Faculty of Mathematics and Applied Physics, Rzeszów University of Technology \\ W. Pola 2, 35-959 Rzeszów, Poland \\ ${ }^{b}$ Institute of Molecular Physics, Polish Academy of Sciences, M. Smoluchowskiego 17, 60-179 Poznań, Poland
}

(Received December 4, 2008; revised version March 24, 2009; in final form April 8, 2009)

\begin{abstract}
The paper presents the results of the share viscosity $(\eta)$ investigations performed for binary mixtures composed of the liquids of extremely different viscosities: a high-viscous $\alpha$-tocopherol acetate $(\eta \approx 10 \mathrm{~Pa} s)$ is mixed with 4 - $n$-propylcyclohexyl- $4^{\prime}-n$-pentylphenyl $(\eta \approx 10 \mathrm{mPa} \mathrm{s})$ and mesitilene $(\eta \approx 1 \mathrm{mPa} \mathrm{s})$, the two low-viscous solvents composed of the non-polar molecules of an essentially different shape. It was found that the viscosities of the mixtures, disregarding the molecular shape of the non-polar admixture, exhibit a strong nonlinear dependence on tocopherol mole fraction and, at a given temperature, the dependence can be described with an exponential function. For a constant tocopherol concentration in the mixtures, the viscosity temperature dependences are well described with the Vogel-Fulcher-Tammann relation. The results can be considered as a model of the viscosity behavior related to the laminar flow of miscible liquids of a different viscosity being in contact to each other.
\end{abstract}

PACS numbers: $65.20 .-\mathrm{w}, 66.20 . \mathrm{Ej}, 83.85 . \mathrm{Vb}$

\section{Introduction}

In numerous chemical processes two (or more) miscible liquids are in contact to each other, so a diffusive controlled interface with the concentration gradients of the both liquids is formed. An extent of this interface area depends on many factors, mainly on the mutual miscibility of the liquids, the time of the contact persistence and the temperature at which the process occurs. One of the most important problems in that field concerns the situation when the two liquids under consideration are in a laminar flow $[1,2]$. That type of flow ensures that the mixing process between liquids streams being in contact occurs through the diffusion only. Such a situation occurs in advanced chemical technologies based on the microfluidics systems $[3,4]$. The fundamental question which arises here is the viscosity behavior within the boundary diffusive area [4]. In general, the problem is not easy to solve, mainly due to an expected complex profile of the concentration gradients and the temperature factor which can strongly modify both the concentration gradient profiles and the viscosity values.

Still, in modern microfluidic devices not only the laminar flow of multiple liquids streams can be realized and precisely controlled but also, in contrast to the conventional methods, the concentration gradients profile generated perpendicular to the flow direction is stable and can be maintained over long period, the effect which is in practical use $[5,6]$.

\footnotetext{
* corresponding author; e-mail: swiergiel@ifmpan.poznan.pl
}

The viscosity behavior in the binary system with the concentration gradients (like in the diffusive controlled interface) can be modeled on the basis of the experimental results of the viscosity measurements performed for solutions of two liquids in question in the whole range of their concentrations. Indeed, as within the diffusive area the liquids mole fractions are changing from 1 to 0 , so the data obtained for solutions in the whole concentration range can represent the situation in that area, however, with one important condition. The condition concerns the concentration gradient profile within the diffusive area. The viscosity data related to studies of the solutions, can model only the case of the linear concentration change within the diffusive area. Such a "linear" situation may be realized in the mentioned above stable gradient profile produced in microfluidic devices, at least within the greater part of the diffusive interface thickness [7].

It is obvious that the greater difference in the viscosity of two liquids, the general features of the viscosity behavior in mixtures will manifest themselves more markedly. In this paper we present the experimental viscosity data obtained for the binary mixtures of a liquid of very high viscosity ( $\alpha$-tocopherol acetate, vitamin E) and two liquids of low viscosities, chosen to verify a possible role of the molecular shape in behavior of the resultant viscosity of mixtures.

\section{Experimental section}

The compounds studied: $\alpha$-tocopherol acetate $(\alpha$-TA) was delivered by SERVA (purity $\geq 97 \%$ ) and mesi- 
tilene (M) - by MERCK (purity $\geq 98 \%$ ) and they were used as supplied; 4- $n$-propylcyclohexyl-4'-n-pentylphenyl, $\mathrm{C}_{3} \mathrm{H}_{7} \mathrm{CyPhC}_{5} \mathrm{H}_{11}$ (3CyPh5) was synthesized and purified (purity $\geq 99 \%$ ) at the Institute of Chemistry, Military Technical University, Warsaw, Poland. The chemical structure of the compounds is presented in Fig. 1. The viscosity measurements were carried out for the mixtures $\alpha$-TA $+3 \mathrm{CyPh} 5$ and $\alpha$-TA $+\mathrm{M}$ in the whole concentration range and in the temperature range of $280.15-355.15 \mathrm{~K}$.

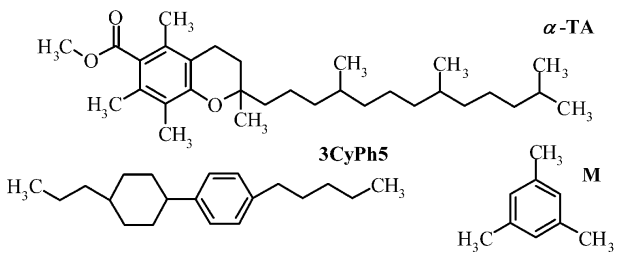

Fig. 1. Chemical structure of $\alpha$-TA, 3CyPh5 and M.

The shear viscosity was measured with a Haake Modular Advanced Rheometer System (MARS) II with a double-cone sensor DC60/1Ti with a small cone angle $\alpha=1.004^{\circ}$. The cone diameter equals $63.564 \mathrm{~mm}$. The gap size between the cones amounts $h=53 \mu \mathrm{m}$. The sensor allows one to measure the torque (resulting from the liquid viscous flow) up to $M_{\max }=0.2 \mathrm{Nm}$, which for the viscosity value of about $10 \mathrm{~Pa} \mathrm{~s}$ corresponds to the limit of the shear rate $D_{\max } \approx 100 \mathrm{~s}^{-1}$ which can be applied. The viscosity values presented in the paper were measured at the shear rate (velocity gradient) of $10 \mathrm{~s}^{-1}$. The temperature was stabilized with the use of a Haake universal temperature controller (UTC) with a Peltier system. Temperature of the samples was stabilized within $\pm 0.1 \mathrm{~K}$. The uncertainty of the viscosity determination was $0.1 \%$.

\section{Results and discussion}

Figure 2 presents the viscosity dependences on $\alpha$-tocopherol acetate mole fraction in the mixtures with two solvent used, whereas Fig. 3 presents, as an example, the viscosity temperature dependence measured for $\alpha$-Tocopherol acetate mole fraction $x=0.78$ in the both solvents. The results presented in Fig. 3 clearly show a secondary importance of the molecular structure of the low viscosity component for the viscosity behavior of its mixtures with a highly viscous $\alpha$-tocopherol acetate.

In Fig. 4 are gathered the experimental viscosity data (points) obtained for all studied mixtures of $\alpha$-TA + $3 \mathrm{CyPh} 5$ and in the whole concentration and temperature range used. The results related to the $\alpha$-TA $+\mathrm{M}$ mixtures are quite similar to those presented in the figure.

Viscosity dependences obtained as a function of both the $\alpha$-tocopherol acetate concentration (at constant temperature) and the temperature (at constant concentration) manifest themselves as a strong (exponential) dependences. At a constant temperature, the viscosity de-

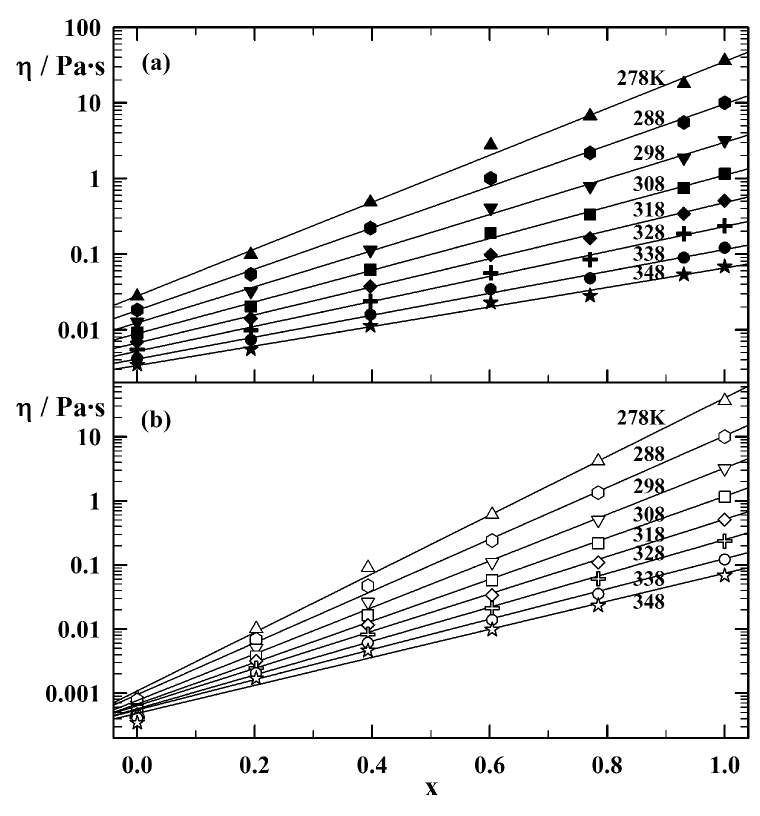

Fig. 2. Viscosity dependences on the mole fraction $x$ of $\alpha$-TA in solutions with $3 \mathrm{CyPh} 5$ (a) and M (b). The solid lines represent the best fit of Eq. (1) to the experimental values (points).

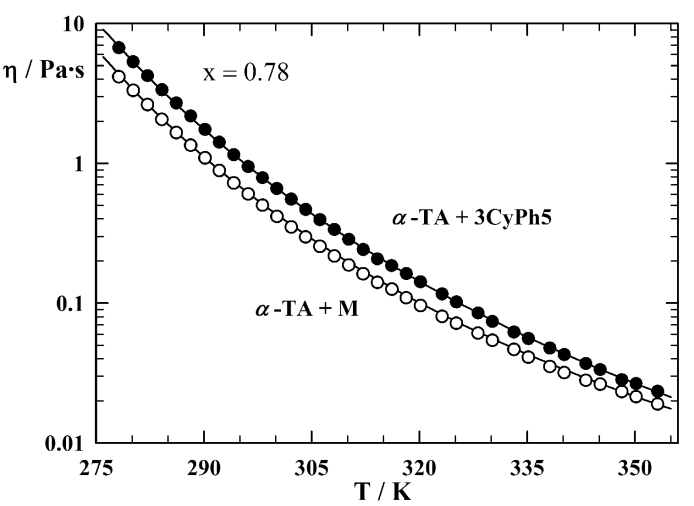

Fig. 3. Temperature dependences of the viscosity of mixtures of $\alpha$-TA $+3 \mathrm{CyPh} 5$ and $\alpha$-TA $+\mathrm{M}$ for $\alpha$-TA mole fraction $x=0.78$. The solid lines represent the best fit of Eq. (2) to the experimental data (points).

pendence on tocopherol mole fraction $(x)$ in the mixtures can be very well reproduced (solid straight lines in Fig. 2 and Fig. 4) with the equation

$$
\ln \eta(x)=\ln \eta(0)+A x,
$$

where $\eta(0)$ is the solvent viscosity, and $A$ is a fit parameter. The values of the parameter $A$ resulting from the best fit of Eq. (1) to the experimental $\eta(x)$ dependences at some different temperatures are presented in Table I. 
TABLE I

The values of parameter $A$ resulting from the best fit of Eq. (1) to the experimental $\eta(x)$ dependences measured for mixtures of $\alpha$-tocopherol acetate + non-polar solvents, at several temperatures.

\begin{tabular}{c|c|c|c|c|c|c|c|c}
\hline \hline & \multicolumn{7}{|c}{$T[\mathrm{~K}]$} \\
\hline$\alpha$-TA+ & 278.15 & 288.15 & 298.15 & 308.15 & 318.15 & 328.15 & 338.15 & 348.15 \\
\hline 3CyPh5 & 7.0371 & 6.2396 & 5.5219 & 4.8411 & 4.3366 & 3.8624 & 3.3697 & 3.0052 \\
M & 10.6653 & 9.4629 & 8.5746 & 7.6927 & 6.9838 & 6.3458 & 5.7103 & 5.3125
\end{tabular}

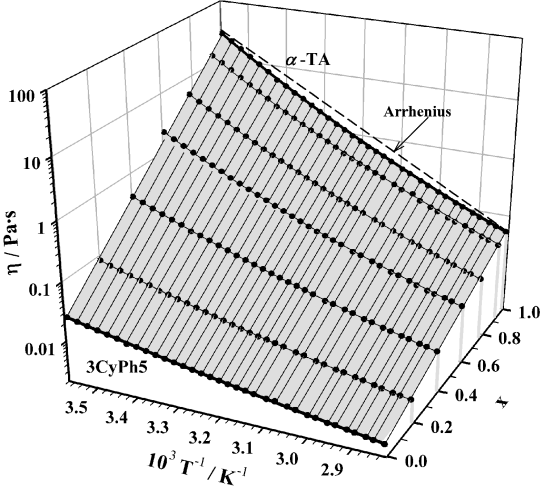

Fig. 4. Temperature and concentration dependences of the shear viscosities measured for mixtures of $\alpha$-TA and $3 \mathrm{CyPh} 5 . \quad x$ denotes the mole fraction of $\alpha$-TA. The solid lines represent the best fit of the equation $\eta(x)=\eta_{0} \exp (A x)$ (at a constant temperature) and the Vogel-Fulcher-Tammann relation $\eta(T)=$ $\eta_{\infty} \exp \left(D /\left(T-T_{0}\right)\right)$ (at a constant concentration) to the experimental data (points).

$\alpha$-Tocopherol acetate belongs to the glass-forming materials $[8,9]$. As presented in numerous papers devoted to that class of materials, the most of their physical properties show an exponential temperature dependence which can be described with the Vogel-FulcherTammann equation $[10,11]$. Indeed, as depicted in Fig. 4 , the temperature dependence of pure $\alpha$-TA viscosity, for instance, exhibits quite important deviation from a commonly used Arrhenius dependence and, as can be also seen in the figure, the Vogel-Fulcher-Tammann equation

$$
\ln \eta(T)=\ln \eta_{\infty}+D /\left(T-T_{0}\right)
$$

reproduces the experimental $\eta(T)$ dependences perfectly (solid lines in Fig. 3 and in Fig. 4). In Eq. (2), $\eta_{\infty}, D$ and $T_{0}$ are the fitting parameters and up to now, their physical meanings are not clear. The obtained values of the parameters are gathered in Tables II and III. Equation (2) was used for description the viscosity temperature dependences also for the mixtures, including pure solvents, where $\eta(T)$ dependences are close to the Arrhenius behavior. It is worthy to be mentioned here that the greater difference in the measured viscosity, the more based is the conclusion on the type of the equation or theoretical model which can be used for description of the dependences obtained. Presented in Fig. 4, the viscosity temperature dependence of $\alpha$-TA, for example, when measured in some narrow temperature range, certainly can be described with an Arrhenius equation, and the slop of the dependence (the so-called activation energy) will be different, depending on the temperature range used.

The results presented in this paper show that the viscosity temperature dependence recorded for the systems studied can be formally described with an alternative way than the Vogel-Fulcher-Tammann Eq. (2). Indeed, as depicted in Fig. 5, the temperature dependence of the coefficient $A$ of Eq. (1), for both solutions studied, fulfills the exponential dependence

$$
A(T)=A_{0} \exp (-B T),
$$

so, together with Eq. (1), the final expression for the viscosity dependence on the solute mole fraction and on the temperature takes a form

$$
\ln \eta(x, T)=\ln \eta(0, T)+x A_{0} \exp (-B T) .
$$

Equation (4) contains two parameters: $A_{0}$ and $B$, both resulting from the experiment. For solutions studied the values of the parameters are following: $A_{0}$ is equal to 175.87 and 211.25 and $B$ is equal to $0.01013 \mathrm{~K}^{-1}$ and $0.01223 \mathrm{~K}^{-1}$ for $\alpha$-TA + mesitilene and $\alpha$-TA $+3 \mathrm{CyPh} 5$, respectively. Equation (4) reproduces the viscosity temperature dependence for different tocopherol mole fractions practically with the same accuracy as the VogelFulcher-Tammann Eq. (2). However, a significance of Eq. (4) is limited to the mixtures of an exponential viscosity dependence on the mole fraction of solute.

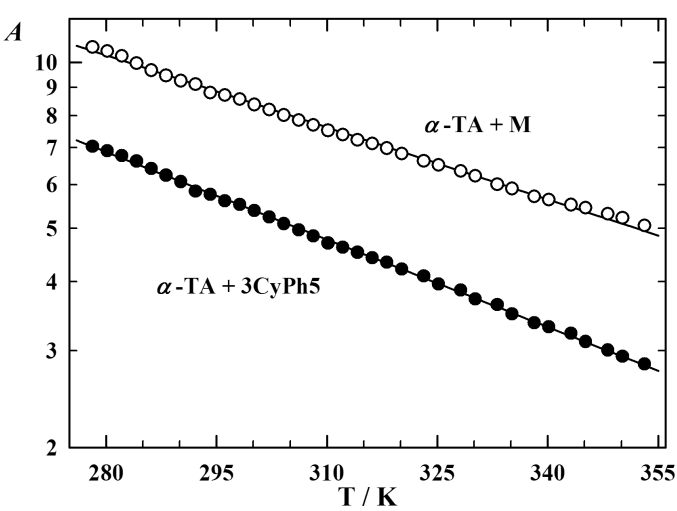

Fig. 5. Temperature dependence of the coefficient $A$ of Eq. (1) for two solutions studied. 
TABLE II

Coefficients of the Vogel-Fulcher-Tammann [Eq. (2)] corresponding to the best fit of the equation to the experimental viscosity temperature dependences recorded for binary mixtures of $\alpha$-tocopherol acetate $(\alpha$-TA $)+3 \mathrm{CyPh} 5 . x$ denotes the mole fraction of $\alpha$-TA in the mixtures.

\begin{tabular}{c|c|c|c|c|c|c|c}
\hline \hline \multicolumn{8}{c}{$\alpha$-TA $+3 \mathrm{CyPh} 5$} \\
\hline & $3 \mathrm{CyPh} 5$ & $x=0.1935$ & $x=0.3969$ & $x=0.6066$ & $x=0.7708$ & $x=0.9115$ & $\alpha$-TA \\
\hline$\eta_{\infty}[\mathrm{mPa} \mathrm{s}]$ & $6.30 \times 10^{-2}$ & $5.07 \times 10^{-2}$ & $2.04 \times 10^{-2}$ & $2.12 \times 10^{-2}$ & $5.47 \times 10^{-3}$ & $6.87 \times 10^{-3}$ & $2.60 \times 10^{-3}$ \\
$D[\mathrm{~K}]$ & 822.3 & 848.8 & 1120.5 & 1176.8 & 1543.8 & 1612.4 & 1872.8 \\
$T_{0}[\mathrm{~K}]$ & 143.2 & 165.3 & 164.8 & 177.9 & 168.2 & 169.4 & 164.5
\end{tabular}

TABLE III

Coefficients of the Vogel-Fulcher-Tammann [Eq. (2)] corresponding to the best fit of the equation to the experimental viscosity temperature dependences recorded for binary mixtures of $\alpha$-tocopherol acetate $(\alpha$-TA $)+$ M. $x$ denotes the mole fraction of $\alpha$-TA in the mixtures.

\begin{tabular}{c|c|c|c|c|c|c}
\hline \hline \multicolumn{7}{c}{$\alpha$-TA $+\mathrm{M}$} \\
\hline & $\mathrm{M}$ & $x=0.2025$ & $x=0.4036$ & $x=0.5972$ & $x=0.7955$ & $\alpha$-TA \\
\hline$\eta_{\infty}[\mathrm{mPa} \mathrm{s}]$ & $9.36 \times 10^{-2}$ & $1.20 \times 10^{-1}$ & $1.20 \times 10^{-1}$ & $4.02 \times 10^{-2}$ & $2.05 \times 10^{-2}$ & $2.60 \times 10^{-3}$ \\
$D[\mathrm{~K}]$ & 237.0 & 473.9 & 582.8 & 889.8 & 1189.4 & 1872.8 \\
$T_{0}[\mathrm{~K}]$ & 171.2 & 170.7 & 190.8 & 185.6 & 182.2 & 164.5
\end{tabular}

The results presented in this paper seem to contain significant information on the momentum transfer process occurring within the boundary diffusive area when two miscible liquids being in a laminar flow, are in contact to each other. In the area where a stable linear (or close to linear) concentration gradient occurs, as produced in many microfluidic devices, the viscosity is changing from one diffusive border to the other one in an exponential way. Of course, the beginning and the ending of the exponential curve of the viscosity evolution in the diffusive area corresponds to the viscosity values of the pure liquids being in the contact. The exponential or close to exponential behavior of the viscosity within the diffusive area is probably one of the main reasons of an advantageous dynamics of two liquid streams flowing in common channel, as observed in the microfluidic devices.

It is also important to realize that the viscosity behavior within the thickness of the diffusive area is very sensible to the temperature changes. The experimental data presented in this paper show that the temperature change is followed by a very strong (exponential) change in the viscosity exponential behavior within the diffusive area.

\section{Acknowledgments}

The viscosity measurements were performed in the Biophysics Laboratory, Department of Physics, Rzeszów
University of Technology. The laboratory has been equipped in the frame of the EU - Polish Integrated Regional Operational Programme (PIROP).

This work was partially supported by the Polish Government grant No. N N2024123 33.

\section{References}

[1] J.C. Giddings, F.J.F. Yang, M.N. Myers, Science 193, 1244 (1976).

[2] J.C. Giddings, Science 260, 1465 (1993).

[3] A. Manz, N. Graber, H.M. Widmer, Sensors Actuators B 1, 244 (1990).

[4] J. Atencia, D.J. Beebe, Nature 437, 648 (2005).

[5] H.B. Mao, P.S. Cremer, M.D. Manson, Proc. Natl. Acad. Sci. USA 100, 5449 (2003).

[6] A. Sawano, S. Takayama, M. Matsuda, A. Miyawaki, Dev. Cell 3, 245 (2002).

[7] N.L. Jeon, H. Baskaran, S.K.W. Dertinger, G.M. Whitesides, L. Van de Water, M. Toner, Nature Biotechnol. 20, 826 (2002).

[8] J.M. Zingg, Mol. Aspects Med. 28, 400 (2007).

[9] K. Kamiński, S. Maślanka, J. Zioło, M. Paluch, K.J. McGrath, C.M. Roland, Phys. Rev. E 75, 011903 (2007).

[10] J. Rault, J. Non-Crystal. Solids 271, 177 (2000).

[11] J.D. Ferry, Viscoelastic Properties of Polymers, Wiley, New York 1980. 